\title{
Rainer Lindner
}

\section{Geschichtswissenschaft und Geschichtspolitik in Weißrußland}

\section{Erinnerungskonkurrenzen in spät- und postsowjetischer Zeit}

Die Umwertung der Geschichte in der Sowjetunion seit Mitte der achtziger Jahre war Teil ihrer Untergangsgeschichte. Kein anderes Feld des öffentlichen Diskurses hat zur Delegitimierung der Sowjetideologie in dem Maße beigetragen wie die offen ausgetragenen Debatten um die Sowjetvergangenheit. Historiker spielten dabei zunächst eine nachrangige Rolle. Das Feld der Geschichte wurde besetzt von Schriftstellern, Regisseuren und Publizisten. Die professionellen Geschichtsforscher fanden erst nach einer Periode der ideologischen Emanzipierung zu einer neuen Sprache. In Weißrußland haben traumatisierende Kollektiverfahrungen wie die Katastrophe von Černobyl' 1986 und die Entdeckung stalinistischer Massengräber 1988 in Kuropaty zur Beschleunigung der historischen Delegitimierung beigetragen. Nach der Erlangung der Unabhängigkeit hat sich indessen aus dem schmalen Bestand gesicherter nationalhistorischer Vergangenheit keine nationale Identität etablieren können. Seit Präsident Aljaksandr Lukašènka im langen Schatten des sowjetischen Erbes das Land regiert, stehen nationalhistorische und russisch-imperiale Interpretationen der weißrussischen Geschichte in harter Deutungskonkurrenz ${ }^{1}$.

1 Ausführlich Rainer Lindner, Historiker und Herrschaft. Nationsbildung und Geschichtspolitik in Weißrußland im 19. und 20. Jahrhundert (Ordnungssysteme. Studien zur Ideengeschichte der Neuzeit 5, München 1999); ders., Weißrußland im Geschichtsbild seiner Historiker, in: Dietrich Beyrau, ders., (Hrsg.), Handbuch der Geschichte Weißrußlands (Göttingen 2001) 25-48; aus weißrussischer Perspektive Henadz Sabanovich, The War Against Belarusian History, in: Education in Russia, The Independent States and Eastern Europe 20 (2002) 1, 18-27; ders. (Henadz' Sahanovič), Dzesjac' hadoŭ belaruskoj historyjahrafii, in: Belaruski Histaryčny Ahljad 8 (2001) 215-229; Dimitri Karev, Aleksandr Nečuchrin, Razvitie istoričeskich issledovanij v Respublike Belarus': obščie tendencii, in: Vesnik Hrodzenskaha dzjaržaŭnaha universitèta, Ser. 1 (2000) 1-15; Aljaksandar Kraŭcèvič, Prablemy histaryčnaj navuki na Belarusi, in: Histaryčny al'manach 4 (2001) 6-13. 


\section{Historiographie als Funktion des Politischen vor 1991}

Die weißrussische Geschichtsschreibung und Erinnerungskultur stand seit 1945 im Zeichen der Kriegserfahrungen im Großen Vaterländischen Krieg. Die Partisanenrepublik, die die größten materiellen und physischen Verluste der gesamten Sowjetunion hinzunehmen hatte, verband ihren Neuaufbau mit einer besonders intensiven ideologischen Aufrüstung. Dem großen Industrialisierungs- und Urbanisierungsschub, den die Weißrussische Sowjetrepublik (Belorusskaja Sovetskaja Socialističeskaja Rèspublika, BSSR) nach 1945 erlebte, entsprach ein hohes wissenschaftlich-technisches Potential der Teilrepublik. Die Zahl der wissenschaftlichen Einrichtungen erhöhte sich zwischen 1956 und 1985 von 76 auf 167. Waren Mitte der fünfziger Jahre 4400 Menschen in wissenschaftlich-technischen und wissenschaftlich-pädagogischen Berufen tätig, gehörten 1985 bereits 42400 Menschen zu dieser Berufsgruppe. Die Zahl der Doktoren der Wissenschaft erhöhte sich dabei von 134 auf 1011 und diejenige der Kandidaten der Wissenschaft von 1600 auf 13100 . Gemessen an der Gesamtbevölkerung gehörte die BSSR bald zu den intelligenzdichten Regionen im östlichen Europa mit einem hohen wissenschaftlichen Potential2. In den sechziger Jahren wurde der Wissenschaftsbetrieb in der BSSR massiv ausgeweitet ${ }^{3}$. Im Vergleich aller Unionsrepubliken nahm lediglich in der Estnischen SSR zwischen 1960 und 1970 die Zahl der Historiker stärker $\mathrm{zu}$ als in Weißrußland. Auf 208\% - von 476 (1960) auf 989 (1970) - wuchs in diesem Zeitraum die Zahl der Geschichtswissenschaftler in der BSSR, wiewohl gleichzeitig der Anteil der Historiker an der Gesamtzahl der Wissenschaftler um 2,44\% auf 4,52\% sank. Gab es 1960 lediglich sechs Doktoren und 165 Kandidaten der Geschichtswissenschaften, verfügte die weißrussische Historiographie zehn Jahre später immerhin über 32 Doktoren und 268 Kandidaten. Im Jahrzehnt zwischen 1970 und 1980 sowie in den letzten Jahren der Sowjetunion hat die $\mathrm{Zu}-$

Tabelle 1: Zabl der Historiker in der BSSR und in der Republik Belarus (1936-2001)

\begin{tabular}{lrrrrrr}
\hline & 1936 & 1960 & 1970 & 1980 & 1988 & 2001 \\
\hline Historiker gesamt & 36 & 476 & 989 & 1082 & 1059 & $?$ \\
Doktoren d.h.W. & $?$ & 6 & 32 & 50 & 62 & 104 \\
Kandidaten d.h.W. & $?$ & 165 & 268 & 457 & 602 & 710 \\
\hline
\end{tabular}

2 Instytut historyi AN Belarusi (Hrsg.), Narysy historyi Belarusi, Bd. 2 (Minsk 1995) 404.

3 Die Zahl der Wissenschaftler in der BSSR nahm von 1960 (6840) bis 1988 (44072) deutlich zu. Das Jahr mit der größten Dynamik war dabei 1962 mit 36\%. Im Überblick Korzenko, Naučnaja intelligencija Belorussii v 1944-1990 gg. (podgotovka, rost, struktura) (Minsk 1995) 67. Zur institutionellen und methodologischen Fortentwicklung der sowjetischen Geschichtswissenschaft vgl. Joachim Hösler, Die sowjetische Geschichtswissenschaft 1953 bis 1991. Studien zur Methodologie- und Organisationsgeschichte (Marburger Abhandlungen zur Geschichte und Kultur Osteuropas 34, München 1995) $15 \mathrm{ff}$., $71 \mathrm{ff.}, 163 \mathrm{ff}$.

${ }_{4}^{4}$ Korzenko, Naučnaja 69-71. 
wachsrate bei den Wissenschaftlern insgesamt und mithin auch bei den Historikern die Werte der sechziger Jahre nicht übersteigen können. Sie ging im Gegenteil immer mehr zurück.

Die Gesamtzahl der verteidigten Doktor- und Kandidatendissertationen belief sich zwischen 1961 und 1970 auf 209, im Vergleich zu 168 in den Jahren 19451960. Erst in den achtziger Jahren ließ das Personalwachstum wieder nach.

War nach 1945 in den Arbeiten der Historiker eine Dichotomie von sowjetpatriotischen und neonationalen Ideologemen zu erkennen, so bildeten sich jetzt und stärker noch nach dem 22. Parteitag der KPdSU vom Oktober 1961 zwei ähnliche Parallelstrategien heraus, die für die Historiographie nicht folgenlos blieben. Einerseits sollte sich die gesellschaftswissenschaftliche Forschung auf „die Praxis des kommunistischen Aufbaus“, deren theoretische Verallgemeinerung sowie auf die „wichtigsten Gesetzmäßigkeiten der wirtschaftlichen, politischen und kulturellen Entwicklung des Sozialismus und seines Hinüberwachsens in den Kommunismus“, wie auch auf die Aufgabe konzentrieren, „das Studium der [... ] Erfahrungen der Kommunistischen Partei und des Sowjetvolkes, der Gesetzmäßigkeiten der Entwicklung des sozialistischen Weltsystems sowie der internationalen kommunistischen und Arbeiterbewegung “ fortzusetzen ${ }^{5}$. Zugleich wurden die „Herausbildung der weißrussischen Nation“, die „nationale Staatlichkeit“ und die „Souveränität der BSSR innerhalb der UdSSR“ nach $1945 \mathrm{zu}$ viel untersuchten Gegenständen der Geschichtswissenschaft ${ }^{6}$. Einer allseits proklamierten „Annäherung und Verschmelzung der Nationen" sowie der Herausbildung eines „Sowjetvolkes“ stand das Interesse an der individuellen Entwicklung der „sozialistischen Nationen“ gegenüber. Der Nationsbegriff selbst hatte sich gewandelt. Gemeint waren damit jetzt Regionalgruppen des Sowjetvolkes, aus deren nationaler Kultur die „veralteten, den Aufgaben des kommunistischen Aufbaus nicht entsprechenden Formen" zu eliminieren waren ${ }^{7}$. Die in der Verfassung der BSSR von 1936 festgehaltenen Attribute der staatlichen Souveränität ${ }^{8}$ blieben indessen formale Zugeständnisse und standen - etwa hinsichtlich des Rechts auf den freien

5 Programm der KPdSU, in: Programm und Statut der Kommunistischen Einheitspartei der Sowjetunion. Angenommen auf dem XXII. Parteitag der KPdSU 17. bis 31. Oktober 1961 (Berlin 1961) 3-134, hier $121 \mathrm{f}$.

6 Vgl. u.a. S. R. Vicharev, Suverenitet BSSR v sostave SSSR (Minsk 1958); Instytut filasofii AN BSSR (Hrsg.), Farmiravanne i razvicce Belaruskaj sacyjalistyčnaj nacyi (Minsk 1958); im folgenden zitiert: Farmiravanne i razwicce; Ja. Karnejčyk, Belaruskaja nacyja. Histaryčny narys (Minsk 1969).

7 N. S. Chruschtschow, Über das Programm der Kommunistischen Partei der Sowjetunion, Oktober 1961, in: ders., Der Triumph des Kommunismus ist gewiß (Berlin 1961) 161-307, hier $251 \mathrm{ff}$.

${ }^{8}$ Die wichtigsten waren das Recht: auf eine Verfassung, auf den freien Austritt aus der UdSSR, keine Gebietsveränderungen ohne Einverständnis der BSSR zuzulassen, auf eine bilaterale Außenpolitik, zur Aufstellung eigener bewaffneter Organe, auf eine eigene Staatsbürgerschaft (republikanskoe graždanstvo), auf eigene Vertretungen in den höchsten Organen der UdSSR, zur Durchführung eines republikweiten Referendums, auf das Einbringen eigener Gesetzesinitiativen in die unionsweite Rechtsprechung sowie auf den Besitz einer Flagge, eines Wappens und einer Hauptstadt. Vgl. Vicharev, Suverenitet BSSR 108-128. 
Austritt aus der Union - in der Tradition der frühsowjetischen Nationalitätenpolitik. Die nationalen Merkmale der Weißrussen kämen deutlich „in den Formen der Wohnarchitektur, in den Ornamenten und Farben der Kleidung, in Traditionen des täglichen Lebens und besonders in der Volkskunst, die organisch mit der Geschichte, der Natur und der nationalen Psychologie des Volkes verbunden sind“, zum Ausdruck9. Immerhin schien die damals übliche Formulierung von der Gleichzeitigkeit des Nationalen und Internationalen gewisse Interpretationsspielräume einzuräumen ${ }^{10}$.

Es galt, die „wichtigsten Vorzüge“ der sozialistischen gegenüber den bürgerlichen Nationen - die "Gleichheit der Völker und Nationen, Liquidierung der Klassenunterschiede sowie die sozialistischen Produktionsbedingungen" - in die Rhetorik einfließen zu lassen ${ }^{11}$.

Dennoch fiel auf, daß neben den vom Moskauer ZK formulierten und allgemein verbindlichen Vorgaben für die Wissenschafts- und Kaderpolitik in den Republiken eigene Akzente gesetzt wurden. Für die weißrussische Historiographie bedeutete dies eine intensive Erforschung des Großen Vaterländischen Krieges und der Partisanenbewegung. Mit K. T. Mazuraŭ stand von 1956 bis 1965 ein ehemaliger Partisan an der Spitze des ZK, der die spezifische Erfahrungslage einer ganzen Generation weißrussischer Politiker dieser Zeit verkörperte. Sein Nachfolger im Amt des Ersten Sekretärs des ZK, P. M. Mašeraŭ, forderte eine Abhandlung zu „Ursprung und Charakter der weißrussischen Partisanenbewegung im Großen Vaterländischen Krieg"12.

Die BSSR-Historiker wurden mit einer „Bringschuld der Historiker Weißrußlands vor dem Volk " konfrontiert ${ }^{13}$. Mit quasinationaler Emphase sollte ein Mobilisierungsschub erfolgen und die ideologische Ausrichtung der Geschichtswissenschaft befördern.

Von 1961 bis 1985 erlebte die weißrussische Geschichtsschreibung ihre bislang produktivste Phase. In dieser Zeit sind sechs Gesamtdarstellungen zur weißrussischen Geschichte in zusammen elf Bänden erschienen, aus denen die bis heute umfangreichste und von über hundert Autoren verfaßte „Geschichte der Weißrussischen SSR“ (1972-1975) herausragt ${ }^{14}$. Gefördert wurden außerdem enzyklopädische Großausgaben mit ausführlichen historischen Artikeln. Fortgesetzt

${ }^{9}$ V. F. Kopytin, Nacional'noe $\mathrm{i}$ internacional'noe $\mathrm{v}$ žizni belorusskogo naroda, in: Mogilevskij obkom KP Belorussii/Oblastnoj metodičeskij sovet prepodavatelej obščestvennych nauk (Hrsg.), Sbornik materialov naučno-teoretičeskoj konferencii „XXIV s”ezd KPSS i social'no-političeskoe razvitie sovetskogo obščestva“ (Gorki 1972) 89-92, hier 92.

10 Vgl. u. a. N. S. Perkin, Nacional'nae i internacional'nae ŭ litaratury (Minsk 1971).

11 Farmiravanne i razvicce 4.

12 P. Mašerov, Idejno-političeskoj rabote - vysokuju dejstvennost' (Moskau 1975) $70 \mathrm{f}$.

13 Za glubokuju razrabotku voprosov istorii BSSR i Kompartii Belorussii, in: Kommunist Belorussii (1960) 72-77, hier 73 .

14 Institut Istorii Akademii Nauk BSSR (Hrsg.), Istorija Belorusskoj SSR. V dvuch tomach. Vtoroe dopolnennoe izdanie (Minsk 1961); Institut Istorii Akademii Nauk BSSR (Hrsg.), Historyja Belaruskaj SSR. U pjaci tamach (Minsk 1972-1975). 
wurde auch die großzügige Herausgabepolitik für Dokumentationen zur vorsowjetischen und Sowjetgeschichte sowie für Chrestomathien gleicher Einteilung ${ }^{15}$. Die wesentlichste Ursache für die „Produktivität“ der weißrussischen Geschichtswissenschaft in den sechziger, siebziger und frühen achtziger Jahren bestand fraglos in der „Stabilität" des Geschichtsbildes, waren doch die Jahrzehnte zwischen 1920 und 1950 von mehr oder minder unterschiedlichen Perspektiven und politischen Grabenkämpfen innerhalb der historischen Forschung geprägt gewesen. Gänzlich ausgeblendet wurden in den nichtrussischen Republiksgeschichten sämtliche antirussischen Töne. Der historische „Kampf des weißrussischen Volkes für eine Wiedervereinigung mit Rußland" blieb ebenso ein bevorzugtes und dankbares Objekt der Untersuchung wie die Partisanen- oder Kriegsthematik ${ }^{16}$. Von dem für die sechziger Jahre beobachteten Distanzzuwachs zwischen Geschichtswissenschaft und Politik und der von Roger Markwick und Joachim Hösler nachgewiesenen und mit den Namen A. I. Danilovs, K. N. Tarnovskijs, Ja. S. Drabkins, A. Nekričs und vor allem M. Ja. Gefters verbundenen „Demokratisierung“ oder „Revision“ des ideologieschweren Geschichtsbildes waren auch in Weißrußland Ansätze zu erkennen. Der Wegfall extremer politischer Bevormundung eröffnete nicht zuletzt für die Quellenarbeit neue Freiräume und ließ sogar wieder nationale Töne anklingen ${ }^{17}$. Themenfelder wie die Geschichte des Druckwesens und des Reformators und Schriftgelehrten Franzišk Skaryna, die Sozialgeschichte der weißrussischen Territorien zwischen dem 16. und dem 18. Jahrhundert sowie die Ethnogenese der Weißrussen sorgten sogar für eine gewisse Konstanz der nationalhistorischen Forschung in der BSSR. In der Art ihrer Bearbeitung spiegeln sich Dauer und Wandel des Geschichtsbildes der weißrussischen Sowjetgesellschaft.

Insgesamt gesehen überwiegen die Anzeichen dafür, daß die weißrussische Geschichtsschreibung bis in die zweite Hälfte der achtziger Jahre über eine funktions- und leistungsfähige institutionelle Infrastruktur verfügte. Die relative Stabilität der Sowjetgesellschaft zog eine nachhaltige Verfestigung des vorherrschenden Geschichtsbildes unmittelbar nach sich. Die Publikationsmenge stieg permanent an. Der Spielraum der Historiker zur Untersuchung von nationalhistorischen Randthemen wurde indessen immer kleiner und führte vereinzelt zum Eklat. Die Geschichtsschreibung der BSSR wie der gesamten Sowjetunion befanden sich in einer „produktiven Lethargie“, aus der sie erst ein politischer Impuls zu befreien vermochte.

15 Die zwölfbändige weißrussischsprachige Enzyklopädie P. U. Broŭka u.a. (Hrsg.), Belaruskaja saveckaja Encyklapedyja, Bde. 1-12 (Minsk 1969 ff.).

16 A. P. Ignatenko, Bor'ba belorusskogo naroda za vossoedinenie s Rossiej. Vtoraja polovina XVII-XVIII v. (Minsk 1974).

17 Hösler, Die sowjetische Geschichtswissenschaft $71 \mathrm{ff}$. Zur „revisonistischen Richtung“ vgl. Roger D. Markwick, Catalyst of Historiography, Marxism and Dissidence: The Sector of Methodology of the Institute of History, Soviet Academy of Science, 1964-1968, in: EuropeAsia-Studies (1994) 579-596. 
Die eigentliche Wende in der sowjetischen Geschichtswissenschaft begann aus der Defensive ${ }^{18}$. Anders als der mit den Chiffren Glasnost und Perestroika verbundene Reformversuch der sowjetischen Führung unter Gorbačev wurde der Richtungswechsel in den Gesellschaftswissenschaften keineswegs von oben forciert. Das auf dem 27. Parteitag der KPdSU angenommene Parteiprogramm warnte die Historiker gar vor einem „Konjunkturrittertum" 19 und war, wie auch Gorbačevs Festvortrag anläßlich des 70 . Jahrestages der Oktoberrevolution, nicht auf eine generelle Umdeutung der Geschichte aus ${ }^{20}$. Es waren die Medien des Films, der Literatur und des Theaters, in denen die ersten Signale zu einer Neubewertung der Sowjetgeschichte gesetzt wurden. Zwischen 1987 und 1989 zogen der Film „Die Reue" des georgischen Regisseurs Tengiz Abuladze, der Roman „Die Kinder des Arbat" von Anatolij Rybakov und die Stücke "Weiter, weiter, weiter" und „Der Brester Frieden" von Michail Šatrov ein neues historisches Interesse breiter Kreise der Gesellschaft und auch der Geschichtswissenschaften nach sich. An der nationalen Peripherie machte in Anlehnung an Ajtmatov der Begriff des „Mankurtismus" Furore, welcher, nach einer Parabel des Epos „Manas“, synonym für den Verlust des historischen Gedächtnisses und die Loslösung von den nationalen und sprachlichen Traditionen während der Sowjetzeit verwandt wurde.

Anders als in Rußland waren es in der BSSR, neben der aufklärerischen Rolle, die auch die Literatur spielte, konkrete Ereignisse, die eine Historisierung der $\mathrm{Ge}-$ sellschaft und den vorläufigen Kurswechsel des Geschichtsdenkens bewirken sollten. Das völlige Totschweigen der kulturellen und sprachlichen Traditionen Weißrußlands im erstarrten Brežnev-System führte seit $1985 \mathrm{zu}$ ersten Wortmeldungen der nationalen Intelligenz. Am 15. Dezember 1985 wandten sich 28 weißrussische Wissenschaftler, Schriftsteller und Künstler in einer Petition an den neuen Generalsekretär Gorbačev und machten auf die Geringschätzung der weißrussischen

18 Zur Orientierung vor allem Mark von Hagen, History and Politics under Gorbachev: Professional Autonomy and Democratization, in: Paul Lerner (Hrsg.), The Soviet Union 1988: Essays from the Harriman Institute Forum (New York 1989) 181-197; T. Ito (Hrsg.), Facing Up to the Past. Soviet Historiography under Perestrojka (Sapporo 1989); Donald Raleigh (Hrsg.), Soviet Historians and Perestrojka (London 1989); Michail Rožanskij, Geschichte: Antworten auf nicht gestellte Fragen, in: Klaus Segbers (Hrsg.), Perestrojka: Zwischenbilanz (Frankfurt a.M. 1990); Henry Kozicki (Hrsg.), Western and Russian Historiography. Recent Views (New York 1993).

19 Der Begriff stammt aus dem Programm der Kommunistischen Partei der Sowjetunion. Neufassung. Angenommen auf dem XXVII. Parteitag der KPdSU, in: XXVII. Parteitag der KPdSU. Dokumente (Moskau 1986) 5-104, hier 75.

20 Michail Gorbatschow, Der Oktober und die Umgestaltung: Die Revolution wird fortgesetzt. Ansprache des Generalsekretärs des ZK der KPdSU, Michail Gorbatschow, auf der Gemeinsamen Festsitzung des Zentralkomitees der KPdSU, des Obersten Sowjets der UdSSR und des Obersten Sowjets der RSFSR anläßlich des 70. Jahrestages der Großen Sozialistischen Oktoberrevolution (in deutscher Sprache zuerst) in: Neues Deutschland 3.11. 1987, 3-7. 
Sprache in Politik, Gesellschaft und Schule aufmerksam ${ }^{21}$. Die Reaktorkatastrophe von Černobyl', kaum vier Monate später am 26. April 1986, wurde nach den opferreichen Jahren des Weltkrieges und der stalinistischen Repressionen als dritte kollektive Vernichtungserfahrung wahrgenommen. Der entstehende nationale Protest besaß hier ebenso wie in Ales' Adamovičs Brief an Gorbačev vom 1. Juni 1986 eine kulturell-historische Dimension. Er formierte sich als eine Art ethnische Schutzbewegung und signalisierte das Erreichen einer Schmerzgrenze. In Entsprechung zur Moskauer Geschichts- und Erinnerungsgesellschaft „Memorial “ riefen Minsker Intellektuelle und Schriftsteller am 18. Oktober 1988 „Matyroloh“, die erste „Weißrussische gesellschaftliche historisch-pädagogische Gesellschaft zur Erinnerung an die Opfer des Stalinismus" ins Leben, zu deren Vorsitzenden der Minsker Archäologe Zjanon Paznjak gewählt wurde²2. Paznjak hatte im Mai 1988 die Ausgrabungen in den Wäldern von Kurapaty bei Minsk geleitet und ihre Ergebnisse am 3. Juni 1988 in der Zeitschrift "Litaratura i mastactva" veröffentlicht. Dieses Ereignis, bei dem Massengräber aus der Zeit des Stalinterrors entdeckt wurden, hat die weißrussische Öffentlichkeit über die Kreise der Intellektuellen hinaus gleichermaßen gelähmt und wachgerüttelt. Tausende nahmen in der Folgezeit an Schweigemärschen und Großdemonstrationen teil. Von der BSSR-Regierung, der Moskauer Zentrale und vor allem der Partei als Sachwalter des stalinistischen Erbes wurde jetzt nicht mehr nur für die Katastrophe von Černobyl', sondern auch für die Verbrechen von Kurapaty Rechenschaft gefordert. Zumindest die aufgeklärte Öffentlichkeit der weißrussischen Hauptstadt, aber auch der übrigen Bezirksstädte, wurde in diesen Monaten vollends politisiert ${ }^{23}$.

An die Opfer der stalinistischen Diktatur in Weißrußland wurde erstmals am 1. November 1987 vor dem Janka-Kupala-Denkmal in Minsk von 200000 Menschen auf einer Massendemonstration gedacht, die auf Initiative der Jugendorganisationen „Talaka“ und „Tutejšie“ zustande gekommen war. Diese Gruppen waren zwei von über neunzig Verbänden, Klubs und Organisationen, die sich in der Hauptstadt - inoffiziell oder registriert - für soziale, politische oder kulturelle Belange der Gesellschaft einzusetzen begannen ${ }^{24}$. Diese Jugendgruppen waren es auch, die sich am 26. Dezember 1987 zum ersten „Reichstag“ (Val'nyj Sojm) in

21 Zur weißrussischen nationalen Bewegung nach 1985 Jan Zaprudnik, The National Consciousness of the Byelorussians and the Road to Nationhood, in: Vitaut and Zora Kipel (Hrsg.), Byelorussian Statehood. Reader and Bibliography (New York 1988); Astrid Sabm, Die weißrussische Nationalbewegung nach der Katastrophe von Tschernobyl 1986-1991 (Münster 1994).

22 Neben „Matyraloh Belarusi“ entstand mit der „Belaruskaja Assacyjacyja achvjar palityčnych represiij" eine weitere nichtstaatliche Organisation. Bis Mitte der neunziger Jahre wurden etwa 600000 Fälle politischer Verfolgungen registriert.

${ }^{23} \mathrm{Zu}$ den Vorgängen in Kuropaty vgl. die bewegende zweisprachige Dokumentation $Z$. Paz'njak u.a. (Hrsg.), Kurapaty. Artykuly, navukovaja spravasdača, fotazdymki (Minsk 21994). Zur Debatte bis 1993: David R. Marples, Kuropaty: The Investigation of a Stalinist Historical Controversy, in: Slavic Review 53 (1994) 513-523.

24 Die Mitgliederzahlen waren freilich gering und gingen nur in Einzelfällen über fünfzig hinaus. 
Palačanka bei Minsk trafen, dessen Bezeichnung expressis verbis an die Ständeversammlung des Litauischen Großfürstentums erinnern sollte. Eine Folgeveranstaltung fand Mitte Januar 1989 in der "Hauptstadt" Wilna (Vilnius) statt, auf der 246 Delegierte 66 informelle Gruppen aus Weißrußland vertraten. Wie schon 1915 geriet in der neuen Schwellenzeit der späten achtziger Jahre der mittelalterliche Großstaat als „brüderliche" Verbindung von Litauern, Polen und Weißrussen in den Blick. Immer wieder, aber wenig erfolgreich wurde seit 1989 von verschiedenen Seiten der staatliche Zusammenschluß der ehemaligen Teilgebiete des Großfürstentums erwogen. Vasil' Bykaŭ, der wenige Jahre später sein Land wegen der politischen Verhältnisse verlassen mußte und 2003 starb, verwies 1989 auf die "historische Bruderschaft", die Polen, Litauer und Weißrussen verbinde. Zahllose Beispiele ließen sich „für unser gemeinsames, wahrhaft brüderliches Leben innerhalb derselben Landschaft, derselben Kultur und sogar desselben Staates anführen. Wenn dies in der Morgendämmerung der Geschichte möglich war, warum sollte es nicht auch heute möglich sein." 25

Die neu zu entwerfende nationale Geschichte der Weißrussen hatte vor allem eine nichtrussische zu sein. Daher war es folgerichtig, daß sehr bald das Gebilde des ausgedehnten und multiethnisch komponierten Großfürstentums Litauen in den Blick der Historiker kam. Eine wachsende Kenntnis über den langen Zeitraum gemeinsam verbrachter Geschichte führte zu einer wohlwollenden Wahrnehmung der polnischen, litauischen und ukrainischen, eben nichtrussischen Nachbarn ${ }^{26}$. Auch die Wiedergründung der Unierten Kirche am 6. August 1990 im Haus des Weißrussischen Schriftstellerverbandes zeigte die Suche nach einer eher rußlandfernen Identität, obwohl ihr Zulauf auch nach der verzögerten offiziellen Registrierung im November 1991 und ganz im Gegensatz zur Ukrainischen Griechisch-Katholischen Kirche eher gering blieb. Auf dem offiziellen Gründungskongreß der "Weißrussischen Nationalen Front“ (Belaruski Narodnae Front, BNF) am 24. und 25. Juni 1989 in Wilna wählte das Führungskomitee der künftigen Oppositionspartei den Oberassistenten des Akademieinstituts für Geschichte, Zjanon Paznjak, zu ihrem Vorsitzenden. Der Programmentwurf der Volksfront suchte ebenso die eindeutige Anbindung an historische Vorbilder wie die Verwendung der Symbolik des Litauischen Großfürstentums sowie der Weißrussischen Volksrepublik von 1918 - die weiß-rot-weiße Flagge und das Reiterwappen Pabonja. Auf ihre Weise suchten die BNF und nach ihr andere weißrussische Parteien die zwingende Ableitung ihrer Existenz und ihrer politischen Absichten in der Nationalgeschichte.

Die Historiker aus Akademie und Universitäten, die sowohl in der Kommunistischen Partei als auch in der Volksfront stark repräsentiert waren, gehörten in der Tat seit $1988 \mathrm{zu}$ den Kommentatoren der Zeitereignisse, wenngleich auch Autodidakten und selbsternannte Spezialisten in langen Zeitungsspalten die Ge-

25 Litaratura i mastactva, 7. 7. 1989, 1.

26 Vgl. u.a. Karl Hartmann, Warschaus Bemühungen um Weißrußland, in: Osteuropa 43 (1993) 853-863. 
schichte zu erklären begannen. Politik und Geschichte traten als aufeinander bezogene Wirkungsfaktoren in eine ständige Nähe. Es ist nicht übertrieben zu sagen, daß das osteuropäische Wendejahr 1989, bezogen auf die Umwertung von Geschichte in der ausgehenden Sowjetunion, in Weißrußland bereits ein Jahr früher stattfand. So intensiv seither politisch aufgeladene Themen der Zeitgeschichte diskutiert worden waren, so deutlich erfolgte seit 1994 die Rücknahme nationalhistorischer Entwürfe und Symbole.

\section{Das Ende der Umwertung: Geschichtspolitik unter Lukašènka}

Die am 5. März 1994 vom Obersten Sowjet angenommene erste Staatsverfassung betonte die „jahrhundertealte Geschichte der Entwicklung der belarussischen Staatlichkeit" ebenso wie „unser unveräußerliches Recht auf Selbstbestimmung "27. Weißrussische Nationalhistoriker wie Valjancin Hryckevič, für den das Großfürstentum Litauen, der Rus’ und Samogitiens vom 12. Jahrhundert bis 1795 ein „unabhängiger" Staat war, hatten bereits während der vierjährigen Vorbereitung des Verfassungsentwurfs auf konstitutionelle Traditionen hingewiesen, wie sie in der Verfassung der Rzeczpospolita vom 7. Mai 1791 verankert waren ${ }^{28}$. Die Historisierung der Politik und des öffentlichen Lebens schritt in dem Maße voran, wie sich die Geschichtsschreibung selbst politisierte. So hatte der Vorsitzende des Obersten Sowjets, Stanislaǔ Šuškevič, in seiner Ansprache zum 3. Jahrestag der Souveränitätserklärung am 27. Juli 1993 in ähnlicher Weise nach historischen Verknüpfungen gesucht, wie es der neue Präsident des Landes, Aljaksandr Lukašènka, zwei Jahre später anläßlich des 50. Jahrestages des Kriegsendes tat. Während Suškevič noch davor gewarnt hatte, die heutige Gesellschaft nach diesem oder jenem Muster der Vergangenheit zu formen oder die Lehren der Geschichte mit den Beziehungen zu den demokratischen Nachbarn zu vermischen, ließen Lukašènkas Ausführungen keinen Zweifel daran, daß der neuen Herrschaftselite andere politische Ziele und ein anderes Geschichtsbild vorschwebten ${ }^{29}$. Am 20. Juli 1994 leistete der erste Präsident Weißrußlands einen Eid auf das Land und sein Volk. Der vom Volk Weißrußlands gewählte Präsident werde ein Garant der weißrussischen Staatlichkeit sein. „Wir erheben keinerlei Anspruch auf irgendwelche Territorien, unabhängig davon, wem sie früher gehörten. Der größte

27 Kanstytucyja Respubliki Belarus'. Prynjata na trynaccataj sessii Vjarchoŭnaha Saveta Respubliki Belarus' dvanaccataha sklikannja 15 sakavika 1994 hoda (Minsk 1994). Zit. nach der deutschen Ausgabe: Informationszentrum bei dem Ministerium für Auswärtige Angelegenbeiten der Republik Belarus (Hrsg.), Verfassung der Republik Belarus. Verabschiedet vom Obersten Sowjet der Republik Belarus am 15. März 1994 (Minsk 1994) 5.

28 Valjancin Hryckevič, Dva pohljady na adnu kanstytucyju, in: Polymja (1993) 178-192, hier 179, 183.

29 My pryidzem da Belarusi! Vystuplenie Staršyni Vjarchoŭnaha Saveta Rèspubliki Belarus' Stanislava Suškeviča pa Belaruskim tèlebačanni i radyè 27 lipenja 1993 hoda, in: Narodnaja Hazeta 29.7.1993, 1. 
Reichtum Weißrußlands ist sein Volk."30 Mit wechselnder Intensität trat Lukašènka seit seinem Amtsantritt für eine Reintegration im Rahmen des ostslawischen Staatendreiecks Rußland-Ukraine-Weißrußland ein und forcierte die Bildung eines Staatenbundes mit Rußland, der Anfang Mai 1997 formal zustande kam $^{31}$. Bereits 1995 ließ er keinen Zweifel zu, daß in den Beziehungen mit dem "großen östlichen Nachbarn Rußland“ auf dem Weg „zu einer slawischen Einheit" ein neues Niveau erreicht worden sei ${ }^{32}$. Die Annäherung an Rußland war neben einer Konsolidierung der eigenen Machtposition das wichtigste Motiv des Lukašènka-Referendums, das am 14. Mai 1995 durchgeführt wurde. Die Gleichstellung der russischen Sprache mit der bisherigen Staatssprache Weißrussisch (83,1\% Befürwortung) gehörte neben der Frage nach einer wirtschaftlichen Integration mit Rußland (82,4\% Befürwortung) zum Komplex der Annäherung an den übermächtigen Nachbarn. Die Ersetzung der bisherigen Flagge und Wappen durch eine um Hammer und Sichel verminderte BSSR-Symbolik $75,0 \% \mathrm{Be}-$ fürwortung) und die Vollmacht des Präsidenten, das Parlament im Fall von Verfassungswidrigkeiten auflösen zu dürfen, dienten dagegen seiner eigenen Machterweiterung; diese wäre ohne den symbolischen Rekurs auf die Sowjetzeit unmöglich gewesen ${ }^{33}$.

Auch der Feiertagskalender im Weißrußland Lukašènkas gehorchte bald anderen Prämissen. Die Durchführung der Parlamentswahl und des Referendums 1995 - kaum eine Woche nach dem in traditioneller Manier und mit großzügigen finanziellen Zuwendungen an die Kriegsveteranen zelebrierten 50. Jahrestag des Kriegsendes - ermöglichte eine Verknüpfung tagespolitischer Interessen mit emotionsreichen historischen Erinnerungen. Die Symbolkraft des Siegestages sollte am Wahltag in Stimmen für den Kurs des Präsidenten umgemünzt werden. Die Feierlichkeiten, die wie auch in den anderen Hauptstädten der GUS-Republiken zwischen dem 7. und 9. Mai stattfanden, ließen die Gedächtniskultur der untergegangenen Sowjetunion wiedererstehen.

In einer zum Himmel schreienden Verhüllung des „Widerspruchs zwischen privatem Elend und amtlichem Pathos" wurde in diesen Tagen auch in Weißrußland an die Größe der untergegangen Union, den Glanz der Waffen und die gefährdeten Beziehungen zu Rußland erinnert ${ }^{34}$. Lukašènka gefiel sich dabei in der

30 Zit. nach Rainer Lindner, Systemwechsel und Staatsbildung in Belarus' und Ukraine. Historische Voraussetzungen, aktuelle Probleme, neue Akteure (Stiftung Wissenschaft und Politik, Ebenhausen 1995) $19 \mathrm{f}$.

31 Ausführlich ders., Präsidialdiktatur in Weißrußland. Wirtschaft, Politik und Gesellschaft unter Lukaschenka, in: Osteuropa 47 (1997) 1038-1152; ders., Besieged Past. National and Court Historians in Lukashenkas Belarus, in: Nationalities Papers 27 (1999) 631-648.

32 Vystuplenie Prezidenta Respubliki Belarus A. G. Lukašenko na toržestvennom sobranii, posvjaščennom 50-letiju Pobedy v Velikoj Otečestvennoj vojne, in: Sovetskaja Belorussija 11. 5. $1995,2$.

33 Respublika, 16. 5. 1995, 1. Eine ausführliche Analyse in: Neue Zürcher Zeitung 16. 5. $1995,2$.

${ }^{34} \mathrm{Vgl}$. vor allem Dietrich Geyer, Erblasten und Erinnerungen. Mittel- und Osteuropa fünfzig Jahre nach der deutschen Kapitulation, in: Osteuropa 45 (1995) 395-409, hier $405 \mathrm{f}$. 
Pose des Fortsetzers der Sowjetgeschichte. Als Vorsitzender des alternativen Festkomitees der Opposition macht Vasil' Bykaŭ hingegen vor aller Augen deutlich, daß die von der offiziellen Jubiläumsrhetorik geadelten Veteranengenerationen nunmehr „rechtlose Schabracken mit miserablen Renten“ seien, deren einziges Privileg noch darin bestehe, den städtischen Nahverkehr kostenlos benutzen zu dürfen. In seiner scharfen Replik „Der bittere Geschmack des Sieges“ wies Bykaŭ zudem darauf hin, daß die Geschichte der zivilen Opfer des Partisanenterrors erst noch geschrieben werden müsse. Lukašènka richtete starke Beschuldigungen gegen Bykaŭ, die Volksfront und deren nationales Unabhängigkeitscredo, aber auch gegen Stimmen wie Svetlana Aleksievič, deren Prosa und Publizistik die plakative Verarbeitung des Krieges in der sowjetischen und nachsowjetischen Gesellschaft immer wieder kritisiert hatte. Wenige Monate nach den Festveranstaltungen beklagte sie, daß „wir immer den Menschen in Waffen geliebt haben“ und im Leben nie etwas anderes taten, als „Kriege zu führen oder der Kriege zu gedenken“35.

Die Spaltung in ein nationales und ein anationales Geschichtsbild hat zu einer geteilten Erinnerungskultur und zur Aufstellung von getrennten Jubiläumskalendern geführt. Seitdem die Verfechter eines nationalen und antirussischen Geschichtsverständnisses 1994 und 1995 erstmals des Sieges des „litauisch-weißrussischen Truppenverbandes“ bei Orša gegen die Moskauer Eindringlinge im Jahr 1514 gedachten, zogen auch in den Folgejahren Anfang September mehrere Tausend Menschen durch die Straßen von Minsk. Anläßlich der 930-Jahrfeier der Stadt Minsk im Frühjahr 1997 formierte sich am 2. März ein Jubiläums- und Protestzug von 5000 Menschen. An die Gründung der BNR am 25. März 1918 wollten am Jubiläumstag im März 1997, eine Woche vor der Unterzeichnung eines „Vertrages über die Union Rußlands und Weißrußlands“, weit über 10000 Menschen erinnern. Hier und bei anderen Gelegenheiten entsprach es der engen Verbindung historischer und tagespolitischer Motive, daß die Erinnerungsdemonstrationen in Kundgebungen gegen Lukašènka und seine prorussische Orientierung umschlugen. Demonstrationsredner beschuldigten den Präsidenten, unter Verfassungsbruch die Selbständigkeit und die Staatlichkeit Weißrußlands an Rußland abzutreten ${ }^{36}$.

Das Jahr 1996 mußte in der Wahrnehmung der Nationalhistoriker als „Jahr der antiweißrussischen Jubiläen“ erscheinen: Nicht nur, weil die Ständeversammlung der Rzeczpospolita vor genau 300 Jahren, am 29. Dezember 1696, beschlossen hatte, künftig nsämtliche Entscheidungen nurmehr in polnischer Sprache aufzuzeichnen “ und einhundert Jahre zuvor (1596) durch die Brester Kirchenunion die

Außerdem Astrid Sabm, Kein politischer Frühling in Belarus. Das Scheitern der Parlamentswahlen im Mai 1995 und die Verselbständigungstendenzen der Exekutive, in: Osteuropa 45 (1995) 1021-1033, hier 1024f.

35 Vgl. Svetlana Aleksievič, „Nas tak dol'go učili ljubit' čeloveka s oruž'em“, in: Izvestija vom 29. 2. 1996, 3 sowie dies., nJa - ne pisatel' katastrof. Prosto žizn' vokrug nas katastrofična do bessmyslennosti“" in: Narodnaja Hazeta vom 13.4.1996 (Beilage) 7.

36 Der Vertrag zwischen Weißrußland und der Russischen Föderation wurde am 2. April 1997 unterzeichnet. 
Spaltung der orthodoxen Kirche in Polen-Litauen besiegelt worden war. Auch der 10. Jahrestag der Katastrophe von Černobyl' und vor allem das „einjährige Jubiläum des ersten Referendums in der Geschichte des weißrussischen Volkes" gaben im Blick auf die weißrussische Sprache, nach Leanid Lyč, wenig Anlaß zur Freude und rückten das Land immer näher an die Gefahr heran, „eine weiße Krähe - ein Land ohne seine Sprache" zu werden ${ }^{37}$.

Doch nicht nur der Feiertagskalender spaltete seit Mitte der neunziger Jahre das kollektive Gedächtnis der weißrussischen Gesellschaft. Selbst in die zunächst geschichtsfernen Vorgänge der Tagespolitik wurden immer wieder historische Bezüge eingebaut, von denen sich die politischen Akteure eine Mobilisierung der Bevölkerung im Sinne ihrer jeweiligen Interessenlagen erhofften. Vor allem die Parlamentswahlen von 1995, in deren Vorfeld eine informelle Blockbildung zwischen Präsident und konservativen Kräften auf der einen und dem demokratischen Lager mit der Volksfront auf der anderen Seite zu beobachten war, haben diese Absichten zutage treten lassen ${ }^{38}$. Höhe- beziehungsweise Tiefpunkt des Wahlkampfes war der Dokumentarfilm von Jurij Azaronka mit dem programmatischen Titel „Haß. Die Kinder der Lügen“, der am 10. und 12. Mai 1995 im weißrussischen Fernsehen ausgestrahlt wurde. Darin wurde eine demagogische Kampagne gegen die „Weißrussische Volksfront“ geführt, die diese Organisation in eine Reihe mit weißrussischen faschistischen Organisationen aus der Zeit der deutschen Okkupation sowie gegenwärtigen rechten Gruppierungen stellte.

Die Montage von Originalaufnahmen aus den Kriegsjahren mit Äußerungen des BNF-Vorsitzenden Paznjak erinnerte an sowjetische Propaganda schlimmster Provenienz. In gezielter Vorbereitung des anstehenden Referendums wies der Film einmal mehr auf die Benutzung der weiß-rot-weißen Flagge und des Pabonja durch die weißrussischen Kollaborateure während der Besatzungszeit im Zweiten Weltkrieg hin. Die Strategie einer weiteren Diskreditierung der Opposition mittels manipulierter Geschichtsdarstellung sollte tatsächlich aufgehen. Im Ergebnis des Mai-Referendums setzte Lukašènka die Abschaffung der nationalen weißrussischen Symbolik durch. Ein „Gesellschaftliches Komitee zur Verteidigung der weißrussischen nationalhistorischen Symbolik“, das sich in unmittelbarer Reaktion auf diesen, in der postkommunistischen Transformationslandschaft einmaligen Vorgang gebildet hatte, wandte sich in eindringlichen aber erfolglosen Erklärungen an die Bürger des Landes. Was nützten Hinweise auf die Tatsache, daß das Pahonja eines der ältesten europäischen Wappenzeichen überhaupt und bereits in der Schlacht bei Orša von weißrussischen Fähnrichen vorangetragen worden sei, wenn der Präsident des Landes gegen die nationale Erinnerungssubstanz eine ganz andere Gedächtniskultur auszubilden hoffte ${ }^{39}$. Nahezu alle prominenten Nationalhistoriker ohne staatliche Leitungsfunktionen gehörten zu den

37 Leanid Lyč, Trysta hadoŭ nazad belaruskaj move užo vynosili prysud ..., in: Narodnaja Hazeta vom 21. 2. 1996, 3.

38 Die Parlamentswahl von 1995 fand in drei Wahlgängen am 14. Mai, am 28. November und am 10. Dezember statt.

39 Vgl. Viktar Cjarěščanka, Naš dom - Belarus', in: Naše Slovo vom 20. 7. 1996, 1. 
Gründungsmitgliedern des Komitees oder schlossen sich ihm bald an. Vieles spricht dafür, nicht nur von einer Historisierung der Politik, sondern auch von einer Politisierung der Geschichtswissenschaft zu sprechen.

Die Machtrhetorik des Präsidenten teilte sich indessen nicht nur über die Medienpolitik mit, sondern schlug sich in gleicher Weise in seinen öffentlichen Auslassungen nieder. Nur einmal nahm sogar die westliche Öffentlichkeit davon Notiz. Unter Hitler, so bemerkte Lukašènka Ende November 1995 in einer Antwort auf die Frage eines Korrespondenten des „Handelsblattes“ nach einer möglichen Präsidialdiktatur in Weißrußland, habe Deutschland den höchsten Punkt seines jahrhundertlangen Strebens nach Ordnung erreicht: „Dies ist genau das, was unserem Verständnis von einer Präsidentenrepublik und der Rolle des Präsidenten in ihr entspricht. Ich möchte unterstreichen, daß es unmöglich ist, $\mathrm{da} ß$ in einem Menschen alles schwarz oder alles weiß wäre. [...] Hitler schuf ein mächtiges Deutschland dank einer starken Präsidialmacht. [...] Wir durchleben heute einen solchen Zeitabschnitt, in welchem ein Zusammenraufen um einen einzelnen oder eine Gruppe von Leuten nötig ist, um zu überleben, aufzustehen und wieder auf die Beine zu kommen." 40

Wenngleich es unangemessen ist, Lukašènka grundsätzliche Sympathien für den Nationalsozialismus zu unterstellen, offenbarte dieses Interview doch allzu deutlich seinen erratischen Umgang mit Geschichte. Ein geschlossenes Weltbild oder eine eigene Ideologie standen dem Präsidenten hier wie auch später nicht zur Verfügung. Vielmehr ruft er jeweils Versatzstücke der sowjetischen Geschichtspropaganda auf, die er geschickt oder weniger geschickt den Kontexten zuzuordnen vermag. Es zeigte sich sehr schnell, daß Lukašènka, der selbst kurzzeitig als "Geschichtslehrer" in der Provinz gearbeitet hatte, vom späteren Dienst in der Armee und in der Kolchose Erfahrungen als Propagandist mit ins Amt brachte. Nicht ohne charismatische Züge verstand er es dabei, politische Reden oder Aktivitäten unter Verwendung historischer Verweise oder Sprachbilder massenwirksam zu inszenieren ${ }^{41}$. Zum Beraterstab des Präsidenten gehörten nicht umsonst von Beginn an auch Historiker, die ihn mit einer entsprechenden Rhetorik auszustatten versuchten.

Erst nach 1991 erfolgte eine erste korporative Organisation der Historiker in der "Weißrussischen Historikerassoziation“ (BAH), die im Februar 1993 auf Initiative des Instituts für Geschichte an der Akademie ins Leben gerufen wurde. Hier versammelten sich außer der akademischen Intelligenz auch Bibliothekare, Museumsangestellte, Archivare und Lehrer. Die Führungsriege setzte sich indessen nur aus den bekannten Namen zusammen ${ }^{42}$. Dem allgemeinen Rückgang des

40 Nach Svaboda vom 2. 12. 1995, 5.

41 Rainer Lindner, The Lukashenka Phenomenon, in: Independent Belarus. Domestic Determinants, Regional Dynamics, and Implications for the West, ed. by Margarita M. Balmaceda, James T. Clem, Lisbeth L. Tarlow (Cambridge, Mass. 2002) 77-108.

42 Zum Vorsitzenden der Assoziation wurde der damalige Dekan der Geschichtswissenschaftlichen Fakultät der BDU, Petr Šupljak, gewählt, zu seinen Stellvertretern wurden der Direktor des Akademieinstituts für Geschichte, Michas' Kascjuk, und der Direktor des 
Wissenschaftspotentials in Weißrußland folgte naturgemäß auch eine deutliche Abnahme der Mitgliederzahl der BAH.

Die institutionelle Struktur der staatlichen geschichtswissenschaftlichen Forschung und Lehre in Weißrußland hat sich in nachsowjetischer Zeit hingegen nur unwesentlich geändert. Lediglich das „Nationale Wissenschafts- und Bildungszentrum ,F. Skaryna“" (1991) und die an Traditionen der Zwischenkriegszeit anknüpfende „Archäographische Kommission“ beim „Staatskomitee für Archivund Rechtsfragen“ (1999) wurden neu geschaffen. Die wichtigsten wissenschaftlichen Einrichtungen für die historische Forschung in Minsk sind das Institut für Geschichte der Akademie der Wissenschaften, das Institut für Kunstgeschichte, Ethnographie und Folklore der Akademie der Wissenschaften und das Nationale Wissenschafts- und Bildungszentrum „F. Skaryna“. Es bestehen neben der als Leitinstitut geltenden Historischen Fakultät der Weißrussischen Staatsuniversität in Minsk sechs weitere historische Hochschulfakultäten (Hrodna, Homel', Brest, Navapolack, Mahilèŭ, Viciebsk). Obgleich zwischen 1986 und 1996 vor den sechs weißrussischen Prüfungskommissionen 57 Doktor- und 250 Kandidatendissertationen verteidigt wurden, war ein quantitativer Rückgang nach dem Zusammenbruch des sowjetischen Wissenschaftsbetriebes nicht zu übersehen ${ }^{43}$. Der Trend konnte erst Ende der 1990er Jahre gestoppt werden. Zwischen 1999 und 2001 wurden 20 Doktor- und 91 Kandidatendissertationen verteidigt.

In der zweiten Hälfte der neunziger Jahre herrschte kaum noch ein genauer Überblick über die Zahl der in Lohn und Brot stehenden Historikerinnen und Historiker. Fast alle waren an mehreren Institutionen oder Projekten gleichzeitig tätig. Andere wanderten in andere Berufe ab, ohne zuvor ihr Arbeitsverhältnis offiziell zu beenden. 1990 arbeiteten in Weißrußland 62 Doktoren und 602 Kandidaten der Historischen Wissenschaften, zehn Jahre später, Anfang 2000 betrug die Zahl 104 Doktoren und mehr als 700 Kandidaten der Historischen Wissenschaften, wenngleich längst nicht alle ausgebildeten Historiker in ihrem Beruf tätig waren. Am Akademieinstitut arbeiteten Ende 199915 Doktoren und 48 Kandidaten, an der Historischen Fakultät in Minsk waren es 11 Doktoren und $52 \mathrm{Kandi-}$ daten.

Wegen der starken Politisierung des Berufes des Historikers seit Beginn der neunziger Jahre sahen einige Beobachter bereits die „letzten Tage der Historiographie" gekommen ${ }^{44}$. Unter dem Druck der anationalen politischen Entwicklung kam es jedoch zur weiteren Polarisierung der Geschichtsdeutungen. Das Jahr

Weißrussischen Forschungszentrums für Dokumentation, Archäographie und Archivwesen, Uladzimir Michnjuk, ernannt. Der Wortlaut des Statuts der Assoziation findet sich in: Statut Belaruskaj asacyjacyi historikaŭ, in: Belaruski Histaryčnaj Časopis 2 (1994) 6-8. 1997 wurde Šupljak von Kascjuk als Vorsitzender abgelöst.

43 Wie in sowjetischer Zeit wird bislang der akademische Grad eines Doktors der Wissenschaften ausschließlich vom Präsidium des „Obersten Attestierungskomitees“ (VAK) der Republik Weißrußland zuerkannt, während die Kandidaten sich den „Räten zur Verteidigung der Dissertation" zu stellen haben. Die Annahme der Dissertation und ihre erfolgreiche Verteidigung wird dann vom VAK lediglich bestätigt.

44 Aleb Dzjarnovič, Apošnija dni histaryjahrafii, in: Naša Niva (1993) Nr. 15, 11. 
1996 gab in der weißrussischen Geschichtswissenschaft den Auftakt zu Flügelkämpfen in der Historikerschaft. Die Auseinandersetzungen erinnern dramatisch an die Vorgänge der Stalinzeit, als sich Nationalhistoriker der Anwürfe der Partei und der von ihr gelenkten Kollegen zu erwehren hatten. Mit der Polarisierung der politischen Kräfte begann sich die Geschichtsschreibung in mehrere Interessengruppen zu spalten, wobei seit Sommer 1996 der Ton der Auseinandersetzung immer mehr den Schuldzuweisungen und Denunziationen der ausgehenden zwanziger und späten vierziger Jahre zu gleichen schien ${ }^{45}$.

Die Lage spitzte sich zu, als ein Dutzend Minsker Historiker und Geschichtslehrer im Frühjahr 1996 die Weißrussische Republikanische Vereinigung „Historisches Wissen“ ins Leben rief. Die öffentlichen Erwiderungen des ersten Direktors der Organisation, des damals 85jährigen Adam Zaleski (gest. 2002) auf neuere Großpublikationen des Akademieinstituts für Geschichte und der Redaktion „Weißrussische Enzyklopädie" waren ebenso wie ihre apologetischen Auslassungen zur „Rolle des Präsidenten der Republik Weißrußland in der gegenwärtigen sozialökonomischen und politisch-moralischen Situation des Landes" als Loyalitätsbekundungen gegenüber dem Präsidenten zu deuten. Lukašènka begann in dieser Konstellation, die Rolle der Partei einzunehmen. Ebenso wie die jungen Sowjethistoriker der dreißiger Jahre die Partei für ihren „schonungslosen Kampf ${ }^{\text {“ }}$ gegen die nationalistischen Abweichler rühmten, galt den Altideologen um Zaleski seit ihren ersten Verlautbarungen Mitte 1996 der Präsident als einzig zuverlässiger Verteidiger der Interessen des Volkes gegen die „Anhänger des Kapitalismus" und die „national-extremistischen Kräfte“. Genannt wurden die per Referendum erreichte Abschaffung der „feudal-bürgerlichen nationalistischen Staatssymbolik“, die Wiedereinführung der alten Staatshymne „mit der wunderbaren Musik von Nestor Sokolovski“"46.

Die Zaleski-Gruppe profilierte sich als historische Zensurbehörde der neuen Herrschaft und übernahm damit faktisch die Rolle der Wissenschaftsabteilung des ehemaligen Zentralkomitees. Die seit 1993 erscheinende „Enzyklopädie der Geschichte Weißrußlands“ wurde zum Anlaß genommen, die nationale Geschichtsschreibung öffentlich zu brüskieren. Wiederum war es das Institut für Geschichte und neben anderen auch das Akademie- und Redaktionsmitglied der Enzyklopädie, Ihnacenka, die von der Vereinigung für die „Entstellungen und Verfälschungen der Geschichte Weißrußlands in den vergangenen Jahren“ verantwortlich gemacht wurden. Viele der Artikel der Enzyklopädie zeichneten sich danach durch einen „unangenehmen Antisowjetismus“ und eine „offene Russophobie“ aus. Die "Entstellung der Geschichte“ komme vor allem in den Ausführungen „des bekannten Verfälschers“ H. M. Sahanovič zum Ausdruck, in denen eine „Aggressi-

45 Rainer Lindner, Nationalhistoriker im Stalinismus. Zum Profil der akademischen Intelligenz in Weißrußland, 1921-1946, in: Jahrbücher für Geschichte Osteuropas 47 (1999) 187-209.

46 Zajavlenie Belorusskogo respublikanskogo i Minskogo naučnogo ob”edinenij „Istoričeskie znanija “: O pozicijach i roli Prezidenta Respubliki Belarus'v nynešnem social'no-èkonomičeskom i moral'no-političeskom položenii strany, in: Narodnaja Hazeta vom 16. 8. 1996, 2. 
vität des russischen Staates gegenüber dem Litauischen Großfürstentum“ postuliert wird ${ }^{47}$. Festzuhalten ist, daß die Nationalhistoriographie kaum freundlicher mit der Gegenpartei umzugehen pflegte. Auch bei den Akademiehistorikern war von den "Verfälschern“ und „Dilettanten“ die Rede. „Historische Wahrheit", "Objektivität" und die richtige Einsicht in die "Gesetzmäßigkeiten des historischen Prozesses" finde sich allein bei ihnen ${ }^{48}$. Die Auseinandersetzung deckte lange verdeckte Gräben auf. Spätestens seit 1996 erwies sich die weißrussische Historikerschaft wieder als so inhomogen wie um die Mitte der zwanziger Jahre ${ }^{49}$.

Geschichtspolitik war jedoch auch Schulbuchpolitik. Der Beschluß Präsident Lukašènkas vom 16. August 1995, zwei Wochen vor Beginn des neuen Unterrichtsjahres, sämtliche von 1992 bis 1995 erschienenen Lehrbücher und Unterrichtshilfen der humanwissenschaftlichen Fächer aus dem „System der weißrussischen Schulen und Hochschulen zu entfernen“, gehörte zu den bislang deutlichsten Maßnahmen der Lukašènka-Administration zur Verhinderung eines nationalen Geschichtsbildes. Absurd mochte die Entscheidung nicht zuletzt deshalb erscheinen, weil dem weißrussischen Staatshaushalt im Zustand des freien Falls der Wirtschaft ein Zusatzposten von 226 Milliarden Weißrussischer Rubel zur Erarbeitung und Herstellung von 225 neuen Lehrbuchtiteln in einer Gesamtauflage von 19 Millionen Exemplaren zugemutet wurde ${ }^{50}$. Damit hielten die bis zum Ausgang der Sowjetzeit gültigen Lehrbücher wieder Einzug in die Schulklassen Weißrußlands. Seit dem Unterrichtsjahr 1995/96 waren damit übergangsweise drei Lehrbuchgenerationen gleichzeitig in Gebrauch: die spätsowjetischen Geschichtsbücher, die noch immer nicht vollständig ausrangierten weißrussischen Lehrbücher der Jahre 1992-95 und schließlich die ersten Exemplare der neu verfaßten Unterrichtsmittel. Die Konfusion bei Schülern und Lehrern konnte nicht größer sein, zumal alle drei Buchgenerationen, gerade für das Fach Geschichte, von völlig unterschiedlichen Prämissen ausgingen.

47 Arkadz' Žuraŭski, Histaryčnaja praŭda ne pavinna skažacca. Ab sur'èznych nedachopach vydannja „Éncyklapedyja historyi Belarusi“, in: Narodnaja Hazeta vom 1. 8. 1996, 2-3. Vgl. bereits den offenen Brief der Vereinigung an Lukašenka: Otkrytoe pis'mo Prezidentu Respubliki Belarus’ A.G. Lukašenko. „Rabota po vosstanovleniju istoričeskoj pravdy podvergaetsja jarostnomu soprotivleniju so storony nacional-èkstremistov, pretendujuščich na istinu v poslednej instancii“, in: Narodnaja Hazeta vom 19.6. 1996, 3.

${ }_{48}$ Vgl. Leanid Lyč, Čaho damahajucca fal'sifikatary?, in: Narodnaja Hazeta vom 17. 8. 1993, 2; Michas' Kascjuk, „Bez histaryčnych vedaǔ nel'ha paspjachova budavac' nezaležnuju dzjaržavu“, in: Belaruskaja Minuǔščyna 2 (1996) 2-4.

49 Lindner, Historiker und Herrschaft $200 \mathrm{ff}$.

50 Ders. (Rajner Lindnèr), Padručniki historyi ŭ Belarusi, in: Instytut mižnaradnaba dasledvannja škol'nych padručnika ŭ imja Georba Ekerta, Hermanskaja kamisija JUNESKO, Belaruskaja kamisija JUNESKO (Hrsg.), Historyja štodzennasci i pravy čalaveka (Minsk 2000) 47-72. 


\section{Neue Horizonte der weißrussischen Geschichtsschreibung}

Die Politisierung der Geschichte, zog nicht nur einen „Raskol“, eine Glaubensspaltung, unter den Historikern und eine veränderte Publikationspolitik nach sich. Vor allem die Themenschöpfung und -bearbeitung waren einem Paradigmenwechsel ausgesetzt. Dieser war zunächst jedoch nicht von einer Professionalisierung der Geschichtswissenschaft insgesamt begleitet. So wie früher das Brüdermotiv zwischen Weißrussen und Großrussen die Interpretationsdominante der Geschichtskultur bildete, wurde jetzt - wie oben angedeutet - von Seiten der nationalen Intelligenz auf die "historische Bruderschaft" mit Polen und Litauern verwiesen. Die „nationale Konzeption der weißrussischen Geschichte“, die 1994 in einem umfangreichen Sammelband vorgestellt wurde, enthielt außerdem Hinweise auf andere Schlüsselthemen, die eine Umwertung und nicht selten eine nationale Überhöhung erfuhren. Neben der Debatte zur „Ethnogenese der weißrussischen Nation“, die das "baltische Substrat“ in der Entstehungsgeschichte des weißrussischen Ethnos betonte, neben der vielfältigen Deutung des Begriffes „weiß“ im Namen „belaja rus" "gehörten die staatsgeschichtlichen Themen zum neuen Kanon der professionellen und nichtprofessionellen Historiker. So galt fortan das Fürstentum Polack, das aus der Konkursmasse der Kiewer Rus als starkes Teilfürstentum hervorgegangen war, als Ursprungslandschaft „weißrussischer Staatlichkeit". Eine Synthese des Polack-Bildes der neuen Weißrußland-Historiographie, die deren euphorische Elemente der frühen neunziger Jahr auf ein Minimum reduziert und die nüchterne Faktographie in den Vordergrund gerückt hat, boten Mitte der neunziger Jahre T. M. Karobyškina und H. V. Stychaŭ in dem von beiden verfaßten Abschnitt des "Grundrisses der Geschichte Weißrußlands“. Die beiden Autoren, von denen Štychaŭ bereits in sowjetischer Zeit auf Distanzen zwischen russischem und weißrussischem Ethnos hingewiesen hatte, hielten an der Formel von einem „selbständigen Staat auf dem Territorium des nördlichen Weißrußlands“ fest: „Folglich reichen die Anfänge der weißrussischen Staatlichkeit bis zur Herausbildung der Polacker und Turaŭer Fürstentümer (9.-10. Jahrhundert) und später (12.-13. Jahrhundert) der Fürstentümer Hrodna, Navahrudak und Smolensk und anderer zurück. Das erreichte Niveau der sozialen und politischen Entwicklung dieser Staaten wurde zum Fundament der Staatsbildungsprozesse auf weißrussischem Territorium im Verlauf des gesamten Mittelalters. “51

Als zweites großes Themenfeld der neueren Historiographie wurde das Großfürstentum Litauen, das die politische Geographie des ostmitteleuropäischen Raumes vom 13. bis zum 16. Jahrhundert dominierte, wie bereits in der Zwischenkriegszeit als „Kernstück der weißrussischen Nationalgeschichte" reklamiert ${ }^{52}$. Die Historiker widmeten ihm eine schnell wachsende Aufmerksamkeit

51 T. M. Karobyškina, H. V. Štychaŭ, Rannjae sjarèdnjavečča. Pačatak perachodu da klasavaha hramadstva. Uzniknenne belaruskaj dzjaržaŭnasci (VI-peršaja palova XIII st.), in: Narysy historyi Belarusi, Bd. 1 (Minsk 1994) 51-113, hier 104.

52 Eine kritische Analyse aus ukrainischer Perspektive jetzt $N$. V. Ševčenko, Bilorus'ko- 
(1990: 47 Titel, 1992: 183 Titel) und bemühten sich um den Nachweis, daß dieser Flächenstaat eben nicht primär ein litauischer Unterdrückungsraum gewesen sei, wie es die Geschichtsbetrachtung unter Sowjetbedingungen von ihnen zu sehen verlangt hatte; der litauische Anteil an der Gesamtbevölkerung, an Produktionsleistungen und Truppen in demselben habe lediglich 15 bis $20 \%$ ausgemacht ${ }^{53}$. Vier Argumente wurden seither immer wieder ins Feld geführt, die das Überwiegen des slawischen und mithin weißrussischen Kontingents am Staatswesen anzeigen sollen: das territoriale Kerngebiet um Navahrudak, der ersten Hauptstadt des Großfürstentums, ein zur Amtssprache erhobenes Altweißrussisch, die mehrheitlich orthodoxe Bevölkerung sowie die von den altrussischen Kodifizierungen hergeleitete Rechtsprechung. Daneben wurde ein umfangreicher Katalog erarbeitet, der auf den hohen Entwicklungsstand des mittelalterlichen Staates verweisen sollte: die Übernahme des Magdeburger Stadtrechts, die in den Litauischen Statuten von 1529, 1566 und 1588 fixierte „Adelsverfassung“, die bereits „Elemente des Rechtsstaates" enthalten habe, die Agrarreform von 1557, die Grund und Boden in bäuerlichen Privatbesitz überführte und als die „erste bürgerliche Reform in Osteuropa" anzusprechen sei ${ }^{54}$. In der Perspektive der postsowjetischen weißrussischen Nationalhistoriker verfügte kein anderer europäischer Staat des 16. Jahrhunderts über ein vergleichbar hochentwickeltes Rechtssystem wie das „Litauisch-Weißrussische Großfürstentum"55. Am Beispiel der Beurteilung der Entstehung und des Charakters des Großfürstentums kann zugleich gezeigt werden, daß seit Ende der 1990er Jahre eine Versachlichung der historischen Forschung, eine Denationalisierung der Interpretation und insofern eine Professionalisierung der historischen Forschung in Weißrußland zu beobachten ist. Die Arbeit Aljaksandr Kraŭcevičs, der zur neuen Generation weißrussischer Historiker zu rechnen ist, fällt durch ein ausgewogenes Urteil auf. Die Generation des Autors hat einen Teil ihrer Ausbildung nach 1989 absolviert, ist in der Lage, die westliche Forschungsliteratur zu rezipieren und hat Erfahrungen im Ausland sammeln können. In seinem Buch kritisiert der Autor die einseitigen Debatten um die ethnische „Vorherrschaft" im Großfürstentum Litauen. Nach seinen Forschungen sei dieses im 13. Jahrhundert als eine biethnische Staatsbildung an der baltisch-ostslawischen

Litovs'ka deržava: novi konceptual'ni zasady sučasnoï bilorus'koï istoriohrafiï, in: Ukrajins'kyj Istoričnyj Žurnal (1997) 55-67.

53 Diese Zahl bei Adam Mal'dzis in den Begrüßungsworten des Rundtischgespräches vom April 1992, in: Belarus' u vjalikim knjastve Litoŭskim, 6. Pavel' Lojka hat den territorialen Anteil der Ostslawen im Großfürstentum auf neun Zehntel und ihren Bevölkerungsanteil auf acht Zehntel beziffert. Vgl. ders., Mesto Velikogo Knjažestva Litovskogo, Russkogo, Žemojtskogo v sisteme vostočno-evropejskich gosudarstv XIII-XVI vv. (unveröffentlichtes Manuskript).

54 Diese Charakterisierungen sind in dem Lehrbuch zu finden Leanid Lojka, Tamara Lojka, Sučasny pohljad na historyju Belarusi, in: Kryžovy šljach. Dapamožnik dlja vyvučajučych historyju Belarusi (Minsk 1993) 3-56, hier 14.

55 So Michal Tkačoŭ in: Belarus' u vjalikim knjastve Litoŭskim (Belarusistyka, Minsk 1992) 28. 
Kontaktzone am Oberen und Mittleren Neman (Memel) entstanden ${ }^{56}$. Die Bezeichnung "weißrussischer Staat" taucht bei Kraŭcevič nicht mehr auf.

Historische Differenzierung und quellennahe Analyse nahmen im Jahrzehnt zwischen 1991 und 2001 deutlich zu. Das galt für die Geschichte der Frühen Neuzeit und der Neueren Geschichte, aber auch für die Arbeiten im Bereich der Zeitgeschichte. Das 20. Jahrhundert wurde einer gründlichen historischen Revision unterzogen. Arbeiten wie die Materialsammlung zur "Weißrussifizierung " in den zwanziger Jahren, die an der Historischen Fakultät der Minsker Staatsuniversität erarbeitet wurde ${ }^{57}$, bekunden eine neue Nähe zu den Quellen, die für die postnationalistische Historiographie in Weißrußland verbindlich zu werden beginnt. Nicht zuletzt die sensible Periode des Zweiten Weltkrieges hat eine neue Bewertung erfahren. Vernichtungskrieg, Besatzung, Kollaboration und Partisanenkampf werden in ersten Arbeiten nicht länger mehr als getrennte Sektoren einer Sieger- und Opfergeschichte analysiert. Eine neue Qualität erreicht hier die weißrussische Historiographie durch die Einbeziehung westlicher Quellenbestände, die in ausgedehnten Forschungsaufenthalten erarbeitet wurden. Die Analyse der "Wirtschaftspolitik des nationalsozialistischen Deutschland in Weißrußland 1941-1944 im Spiegel der Quellen und der deutschen Historiographie der 1990er Jahre ${ }^{\alpha 8}$ von Sjarhej Novikaŭ hat für die Internationalisierung der weißrussischen Historiographie Maßstäbe gesetzt. Die Quellenkunde selbst ist auf dem Weg zur Professionalisierung. In Handreichungen für Studenten präsentieren Historiker Techniken und Methoden der Quellenbearbeitung. Quellenkritik etabliert sich auf diese Weise in einem Wissenschaftsmilieu, das über Jahrzehnte hinweg Geschichtsschreibung als Verfahren verstand, das feststehende Überzeugungen und ideologische Prämissen durch Quellen nicht zu verunsichern, sondern zu bestätigen hatte ${ }^{59}: 1932$ wurde in der Parteizeitung „Bol'ševik Belorussii“ ein Rezensent einer historischen Arbeit auf die eigentliche Aufgabe von Geschichte hingewiesen. „Der Rezensent hat übersehen, daß Geschichte nicht um der Geschichte willen geschrieben wird, sondern nichts anderes ist, als in die Vergangenheit verlegte Politik, und die Probleme des Klassenkampfes der Vergangenheit nicht scholastisch, unparteilich und ohne Rücksicht auf die Bedingungen des heutigen Tages dargestellt werden können." 60

Für die spät- und postsowjetische Geschichtskultur in Weißrußland sind drei Tendenzen auszumachen: Erstens hat Mitte der Achtziger, vor allem infolge äußerer Bedrohungserfahrung (Černobyl') oder neuer Erkenntnisse über die Sowjet-

56 Aljaksandr Kraŭcevič, Stvarènne Vjalikaha knjastva Litoŭskaha (Rzeszów 2000) 180.

57 Belarusizacyja 1920-ja hady. Dakumenty i matèryjaly. Pad ahul'naj redakcyjaj $R$. P. Platonava i U. K. Koršuka (Minsk 2001).

58 S. Ja. Novikaŭ, Ėkanamičnaja palityka nacysckaj Hermanii ŭ Belarusi 1941-1944 hh.: ahljad krynic i hermanskaj historyjahrafii 1990-ch hadoŭ (Minsk 2002).

59 V.P. Grickevič, C. B. Kaun, S. N. Chodin, Teorija i istorija istočnikovedenija. Posobie dlja studentov gumanitarnych fakul'tetov (Minsk 2000).

60 Nationales Archiv der Republik Belarus: F. 4, v. 21, sp. 365, a. 6-7: N.N.: Nekotorye zamečanija po povodu recensii Gessena, pomeščennoj v "Bol’ševike Belorusi“ (11.11.1932), hier a. 7. 
geschichte (Kuropaty) zeitgleich zu den Prozessen in der Ukraine oder den baltischen Republiken eine Neubewertung von Geschichte stattgefunden, zunächst in den Binnenkreisen der nationalen Intelligenz und seit 1988 in den Debatten der professionellen Historiker. Zweitens stellt für die Dekade nach 1991 Weißrußland einen Sonderfall dar, da, hier die amtliche Geschichtsinterpretation nicht mit der nationalen Konzeption weißrussischer Geschichte zusammengetreten ist. Die Geschichtspolitik in Weißrußland geht nach einem nationalen bis nationalistischen Intermezzo seit 1995 Wege, die eine Rückkehr in sowjetische Interpretationsmodelle bedeuten. Drittens: Seit den späten 1990er Jahren setzte eine Professionalisierung bei zumeist jüngeren Historikern ein, die keine politische Anbindung ihrer Forschung suchten, die den Kontakt zur wissenschaftlichen Außenwelt pflegten und die internationalen Forschungsergebnisse rezipierten. Die Emanzipation von politischen Interpretationsgrenzen gelang dabei oftmals in der Provinz eher als im Wissenschaftszentrum Minsk. Eine nationale Erinnerung der Weißrussen nach Jahrhunderten der staatlichen und kulturellen Fremdbestimmung und einer gebrochenen Nationalgeschichte ist erst im Entstehen ${ }^{61}$. Staat und Nation sind in Weißrußland noch nicht zusammengetreten. Die nationale Gegenerinnerung hat sich bislang nur partiell gegen eine amtliche Erinnerungspolitik und ein verordnetes kulturelles Gedächtnis behaupten können. Ob dies in der Perspektive gelingt, hängt nicht zuletzt davon $\mathrm{ab}$, ob die politischen Eliten den Wert politischer Eigenständigkeit, historischer Traditionen, nationaler Kultur und europäischer Perspektiven Weißrußlands zu gewichten wissen.

61 Rawi Abdelal, Memories of Nations and States: Institutional History and National Identity in Post-Soviet Eurasia, in: Nationalities Papers 30 (2002) 459-484. 